\title{
Evaluación del coeficiente de endurecimiento del acero AISI 1045 deformado por rodillo
}

\author{
T. Fernández ${ }^{* 1}$, E. Fernández; I. Rodríguez ${ }^{1}$ y D. Alcántara ${ }^{1}$ \\ ${ }^{1}$ Instituto Superior Minero Metalúrgico de Moa, Holguín. \\ e-mail: tfernandez@ismm.edu.cu; efguilarte@ismm.edu.cu; irgonzalez@ismm.edu.cu y dalcantara@ismm.edu.cu
}

(Recibido/received: 23-Julio-2011; aceptado/accepted: 28-Noviembre-2011)

\begin{abstract}
RESUMEN
El objetivo del trabajo es determinar el comportamiento del coeficiente de endurecimiento n por la ecuación de Hollomon en muestras cilíndricas de acero AISI 1045, las cuales, después de ser deformadas, se sometieron a ensayos de tracción. Se utiliza un diseño de experimento donde se tienen en cuenta las variables número de revolución (n) con 27, 54 y 110 rev/min, fuerza de compresión (P) de 500, 1500 y 2500 N y avance (S) de 0,075; 0,125 y $0,25 \mathrm{~mm} / \mathrm{rev}$. Finalmente, aplicando el método de regresión se obtuvo un coeficiente de endurecimiento, el cual se aproxima a la linealidad cuando restringimos el cálculo a rangos de deformación elevados. Este coeficiente de endurecimiento se aplica en la ecuación de Hollomon para determinar el nuevo valor de tensión de fluencia y aplicarlo para el cálculo del trabajo mínimo a realizar en un proceso de deformación en frío empleando rodillo simple.
\end{abstract}

Palabras clave. Deformación Plástica; tensión; deformación; endurecimiento.

\begin{abstract}
The objective of the work is to determine the behavior of the hardening coefficient $\mathrm{n}$ by the equation of Hollomon, in cylindrical samples of steel AISI 1045, those which, after the deformed, were subjected to traction rehearsals. He used an experiment design where are kept in mind the revolution number (n) with 27, 54 and $110 \mathrm{rev} / \mathrm{min}$, it compression forces (P) of 500, 1500 and $2500 \mathrm{~N}$ and feed (S) of 0.075; 0.125 and $0.25 \mathrm{~mm} / \mathrm{rev}$. Finally, applying the regression method a hardening coefficient was obtained, which approaches to the linearity when restrict the calculation to high ranges of deformation. This hardening coefficient is applied in the equation of Hollomon for determine the new value of flow tension and to apply in the calculation of the minimum work to carry out in a cold process of deformation using simple roller.
\end{abstract}

Keywords: Plastic deformation; stress; deformation; hardening.

\footnotetext{
${ }^{*}$ Autor para la correspondencia
} 


\section{INTRODUCCIÓN}

La descripción del comportamiento de la curva esfuerzo - deformación y del endurecimiento que experimenta un metal, a través de expresiones matemáticas, es de suma importancia en diferentes aspectos de su estudio elasto plástico, debido a que la zona plástica de la curva depende de parámetros que predicen los mecanismos de formabilidad y deformación del material (Aparicio et al., 2007).

En los procesos de conformado por deformación plástica son cada vez más requeridas mayores reducciones de dimensiones de los materiales metálicos trabajados en frío o en caliente. Para satisfacer ese requerimiento es imprescindible disponer de soluciones matemáticas que permitan evaluar el efecto de la cantidad de deformación sobre el endurecimiento que se experimenta en el material. En el año 1735 Bülfinger hizo una proposición de tal solución y después, en la primera mitad del siglo pasado realizaron su aporte otros autores (Hollomon, 1945; Ludwik, 1909 y Voce, 1948).

Ono (1972) considera que muchos de los modelos empleados, aunque representan diferentes relaciones empíricas, no posee ningún sentido físico conocido, proporciona mejores aproximaciones a valores experimentales. Entre estos modelos se encuentran los de Ludwik y Swift, los cuales incluyen el efecto de endurecimiento del material por trabajo en frío (Hill, 1998; Jonson y Mellor, 1973).

Una de las ecuaciones más empleada para la determinación del coeficiente de endurecimiento n, es la ecuación de Hollomon, la cual es una ecuación potencial que se expresa

$\sigma=K \cdot \varepsilon^{n}$

Donde

$\sigma-\quad$ Esfuerzo real, $\mathrm{MPa}$

$\varepsilon$ - Deformación real

$\mathrm{K}$ - Constante que define el coeficiente de endurecimiento

$\mathrm{n}-\quad$ Exponente del endurecimiento.

El coeficiente de endurecimiento $\mathrm{n}$, fue precisado por Low, (Low, 1949), quien obtuvo $\mathrm{n}=\varepsilon \mathrm{u}$, siendo $\varepsilon u$ la cantidad de deformación real correspondiente a la resistencia última real $\sigma u$.
La ecuación de Hollomon, aunque es de carácter empírico, permite representar la trayectoria de la curva real $\sigma$ vs $\varepsilon$ en el segmento correspondiente a la deformación plástica uniforme de una probeta durante el ensayo de tracción uniaxial.

Dentro de los modelos constitutivos para metales se encuentra el ensayo de tracción simple, en el cual cuando se ha desarrollado la estricción, se alcanzan grandes deformaciones y grandes rotaciones. Por esta razón, para obtener resultados realistas es necesario emplear elementos con una descripción de la cinemática no lineal, y modelos constitutivos de plasticidad con grandes deformaciones.

El modelo elastoplástico de Von Mises, se emplea habitualmente para representar el comportamiento de metales. También se han propuesto modelos más complejos para plasticidad de metales, por ejemplo aquellos en los que el endurecimiento depende del camino de carga (Ortiz y Popov, 1983), pero la dificultad de los ensayos experimentales necesarios para su caracterización los han hecho poco utilizados.

El ensayo de tracción en metales dúctiles pasa por dos etapas desde el punto de vista del estado tensional de la probeta. En la primera, las deformaciones son pequeñas sin que exista una disminución apreciable de la sección transversal, y el estado tensional en el cuello es uniaxial con distribución homogénea de las tensiones.

La segunda fase se presenta cuando la tasa de aumento de la tensión debido al endurecimiento del material es menor que la tasa con que disminuye la sección transversal. Entonces se presenta un fenómeno de inestabilidad de tipo geométrico, en el que las deformaciones se concentran en la sección central de la probeta, formándose un cuello o estricción (Norris et al., 1978).

El flujo plástico queda confinado en esta zona, permaneciendo en estado de carga plástica, y el resto de la probeta queda en descarga elástica.

Esta segunda fase se caracteriza porque aparecen tensiones radiales y circunferenciales que dan lugar a un estado tensional triaxial no homogéneo en la zona del cuello (Goicolea, 1996). 


\section{METODOLOGÍA}

Para realizar ensayo de tracción, se empleó una máquina CRITM DNS 200, que presenta una carga de $200 \mathrm{kN}$, luego del proceso de deformación, se tomaron medidas del diámetro del cuello en la sección central, con un calibre Vernier de precisión $\pm 0,05 \mathrm{~mm}$.

Se escogieron 10 probetas de acero AISI 1045, con longitud de $120 \mathrm{~mm}$ y de diámetro $11 \mathrm{~mm}$ Norma ASTM E 646 - 00; Goicolea et al., 1996, una sin deformar y nueve deformadas por rodadura para luego ser traccionadas (Altenberger, 2003 y García-Mateo et al., 1998).

Para comprobar la idoneidad del método y los modelos propuestos se desarrolló el tratamiento estadístico de los resultados, tanto los simulados como los experimentales, se realizaron con la utilización del tabulador Microsoft Excel 2007, lo que permitió establecer la necesaria correspondencia entre las observaciones teóricas y las experimentales.

Las muestras fueron deformadas con fuerzas de $500 ; 1$ 500 y $2500 \mathrm{~N}$, con número de revolución 27; 54 y 110 $\mathrm{rev} / \mathrm{min}$ y avance de 0,$075 ; 0,125$ y $0,25 \mathrm{~mm} / \mathrm{rev}$.

Para el ensayo de tracción, se escogieron 28 probetas de acero AISI 1045, con longitud de $120 \mathrm{~mm}$ y de diámetro 11 mm según las normas ASTM E 646 - 00 y ASTM E 8. Se seleccionaron nueve muestras de las 27 analizadas, que según González-Castellanos (2000) y Zlokarnik (2002), este procedimiento se realiza cuando la población seleccionada, representa el $30 \%$ o un por ciento superior con respecto al total de las muestras estudiadas.

La matriz de planificación del experimento aparece reflejada en la tabla 1 .

Para una ecuación constitutiva, la ecuación 2 permite calcular el exponente por endurecimiento de la forma logarítmica de la curva de tensión de esfuerzo verdadero contra deformación verdadera dentro del rango plástico, la carga axial impuesta a partir únicamente del diámetro del cuello, ha sido sugerida en trabajos previos (GarcíaGarino et al., 1999; ASTM E 646 - 00; Novillo et al., 1999; Valiente, 2001)
Tabla 1. Matriz del experimento.

\begin{tabular}{|l|l|l|l|}
\hline $\mathrm{M}$ & $\mathrm{P}(\mathrm{N})$ & $\mathrm{n}(\mathrm{rev} / \mathrm{min})$ & $\begin{array}{c}\mathrm{S} \\
(\mathrm{mm} / \mathrm{rev})\end{array}$ \\
\hline 1 & 500 & 27 & 0,075 \\
\hline 2 & 500 & 54 & 0,125 \\
\hline 3 & 500 & 110 & 0,25 \\
\hline 4 & 1500 & 27 & 0,075 \\
\hline 5 & 1500 & 54 & 0,125 \\
\hline 6 & 1500 & 110 & 0,25 \\
\hline 7 & 2500 & 27 & 0,075 \\
\hline 8 & 2500 & 54 & 0,125 \\
\hline 9 & 2500 & 110 & 0,25 \\
\hline
\end{tabular}

$\log \sigma=\log K+n \log \varepsilon$

Al obtener los logaritmos esfuerzo contra deformación verdadera de la ecuación, de $\operatorname{los}$ datos $(\log \sigma, \log \varepsilon)$, se calcula mediante regresión lineal del Log $\sigma$ contra $\log \varepsilon$, la pendiente, $\mathrm{n}, \mathrm{y}$ el $\%$ de error de la curva.

La ecuación 3 se emplea para calcular la regresión lineal del coeficiente de endurecimiento $n$.

$$
n=\frac{\left[N \sum_{i=N}^{N}\left(\log \varepsilon_{i} \log \sigma_{i}\right)-\left(\sum_{i=N}^{N} \log \varepsilon_{i} \sum_{i=N}^{N} \log \sigma_{i}\right)\right]}{N \sum_{i=N}^{N}\left(\log \varepsilon_{i}\right)^{2}-\left(\sum_{i=N}^{N} \log \varepsilon_{i}\right)^{2}}
$$

Donde $\mathrm{N}$ es el número de datos. Para calcular la pendiente de la curva hecha en forma conveniente y por representaciones simbólicas para facilitar el trabajo con la metodología es:

$Y=\log \sigma \quad \mathrm{N}$ - número de datos pares.

$X=\log \varepsilon$ SD- desviación estándar de n valores.

$b=\log K \quad \mathrm{n}-$ valor de $\mathrm{n}$

Para calcular la pendiente de la linea de regresión lineal que provee el exponente de deformación se muestra en la ecuación 4 de la siguiente forma:

$n=\frac{N \sum X Y-\sum X \sum y}{N \sum X^{2}-\left(\sum X\right)^{2}}$

Y el coeficiente de resistencia se determina por las ecuaciones 5 y 6 . 


$$
\begin{aligned}
& b=\frac{\sum Y-n \sum X}{N M} \\
& K=\exp [b]
\end{aligned}
$$

M - Número de muestras de cada ensayo.

Para determinar a $\varepsilon$, que es la deformación verdadera del logaritmo natural de la relación de la longitud de calibración instantánea (L), a la longitud de calibración original (L0), se tiene en cuenta la ecuación 7 de la forma siguiente:

$$
\varepsilon=\ln \frac{l}{l_{0}} \text { ó } \varepsilon=\ln (1+e)
$$

Siendo ln el logaritmo natural en las condiciones de establecimiento de la curva

$$
\text { e-deformación ingenieril. }
$$

Para la determinación de los valores experimentales de la tensión verdadera cuando no ha ocurrido el acuellamiento se emplea la ecuación 8

$$
\sigma=S(1+e)
$$

Siendo S la tensión ingenieril.

La tensión ingenieril.es donde el material aún no ha sido deformado, es ese instante de tiempo en el cual se comienza a aplicar la fuerza a la probeta sin existir aún la deformación.

Cuando el material tiene trabajo en frío, la deformación ingenieril se puede determinar teóricamente utilizando la ecuación del material de la siguiente manera

$$
e=\frac{\Delta L}{L_{0}}
$$

\section{RESULTADOS Y DISCUSIÓN}

El comportamiento del coeficiente de endurecimiento $n$ se obtiene en correspondencia a los parámetros empleados en cada muestra y según la dureza obtenida, los valores representados en la tabla 2 son válidos hasta el límite de rotura.
Como se observa en la tabla anterior, con el incremento del trabajo en frío el coeficiente se reduce ya que disminuye la capacidad de deformación plástica por el incremento de la deformación, este comportamiento justifica la capacidad de endurecimiento del acero AISI 1045 por rodadura en frío, los valores obtenidos tienden a 0 , esto demuestra que el material se comporta idealmente de forma plástica.

Tabla 2. Resultados del coeficiente $\mathrm{n}$

\begin{tabular}{|l|l|l|l|c|}
\hline $\mathrm{M}$ & $\mathrm{P}(\mathrm{N})$ & $\begin{array}{c}\mathrm{n} \\
(\mathrm{rev} / \mathrm{min})\end{array}$ & $\begin{array}{c}\mathrm{S} \\
(\mathrm{mm} / \mathrm{rev})\end{array}$ & $\mathrm{n}$ \\
\hline 1 & 500 & 27 & 0,075 & 0,11 \\
\hline 2 & 500 & 54 & 0,125 & 0,13 \\
\hline 3 & 500 & 110 & 0,25 & 0,12 \\
\hline 4 & 1500 & 27 & 0,075 & 0,11 \\
\hline 5 & 1500 & 54 & 0,125 & 0,12 \\
\hline 6 & 1500 & 110 & 0,25 & 0,12 \\
\hline 7 & 2500 & 27 & 0,075 & 0,08 \\
\hline 8 & 2500 & 54 & 0,125 & 0,08 \\
\hline 9 & 2500 & 110 & 0,25 & 0,06 \\
\hline
\end{tabular}

Análisis del esfuerzo real último y el coeficiente de resistencia del material

A partir de la base de datos de los ensayos de tracción se obtuvieron los resultados del comportamiento del coeficiente de resistencia (k). En la figura 1 se establece el comportamiento del coeficiente $\mathrm{K}$ en correspondencia a la fuerza aplicada.

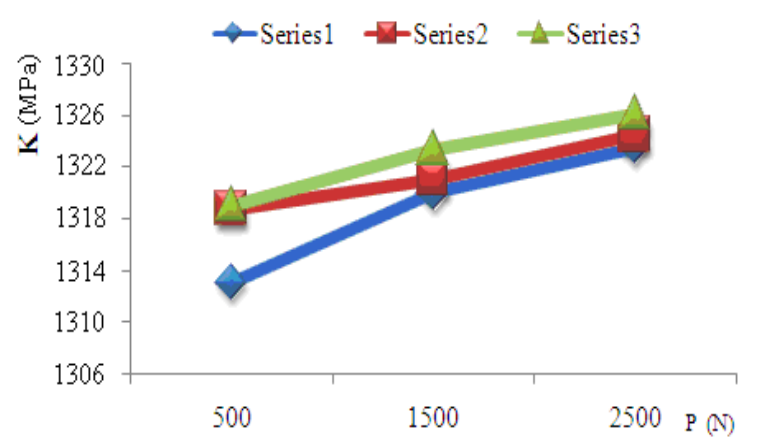

Figura 1. Coeficiente de resistencia (k) en relación a la fuerza.

Callister (1999) plantea que el coeficiente K para los aceros de medio contenido de carbono en estado de recocido es $1250 \mathrm{MPa}$, en la figura 1 el coeficiente de resistencia $\mathrm{K}$ incrementa desde $1312,01 \mathrm{MPa}$ hasta 1 325,52 MPa para las distintas fuerza, avance y número de revolución, como consecuencia de estos ensayos se 
han obtenido diferentes $\mathrm{K}$ según se han introducido los valores durante el proceso de endurecimiento del acero AISI 1045 por rodillo simple.

Las curvas, si bien siguen la misma trayectoria y alcanzan prácticamente la carga máxima, muestran distintos rangos para la variable dependiente, este efecto se debe a que el tamaño de la imperfección influye en el valor final de la fuerza y, consecuentemente en el valor final de la deformación.

La curva tensión nominal - deformación, mediante el ensayo de tracción se comporta de distintas formas para los diferentes materiales, en la figura 2 se muestra el comportamiento del esfuerzo verdadero $(\sigma)$ del acero AISI 1045 deformado por rodadura.

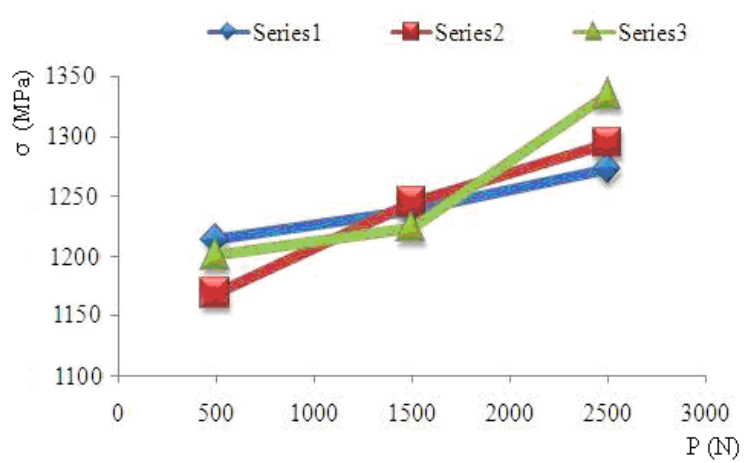

Figura 2 Comportamiento del esfuerzo verdadero del acero deformado y traccionado.

Como se muestra en la figura 2 en las muestras deformadas y luego traccionadas, la tensión se va incrementando en la medida que se introduce mayor fuerza. Existe una tendencia lineal en la medida que se varían los parámetros del proceso, el estado tensional es uniaxial con distribución homogénea de las tensiones.

$\mathrm{Al}$ ir incrementando los valores de la fuerza existe un aumento de la tensión debido al endurecimiento del material, el flujo plástico queda confinado en esta zona, permaneciendo en estado de deformación plástica ya que aparecen tensiones radiales y circunferenciales que dan lugar a un estado tensional no homogéo en el material.

\section{Análisis del esfuerzo ingenieril para el límite de fluencia}

El acero AISI 1045, normalizado y sometido a esfuerzo de tracción experimenta una reducción del área más severa con respecto a un acero de este mismo tipo que ha sido tratado superficialmente, ya que en la superficie del mismo se ha endurecido por la acritud.

La figura 3 muestra el comportamiento del esfuerzo ingenieril con relación a la fuerza aplicada.

Esta tensión mantiene un comportamineto uniforme en dependecia de la carga aplicada como se muestra en las tres series repesentadas.

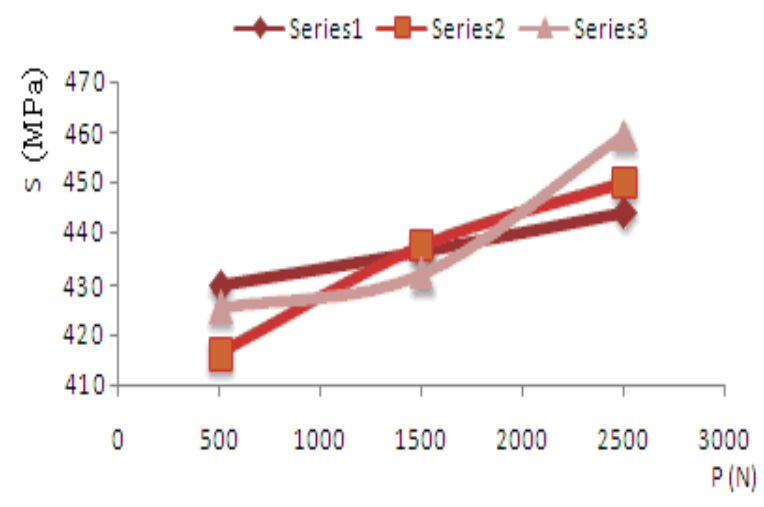

Figura 3. Comportamiento de la tensión ingenieril frente a la fuerza aplicada.

Como se puede observar en cada una de las fuerzas aplicadas por separdo se incrementa el endurecimiento de la pieza durante el proceso de deformación plástica el cual se realiza de forma gradual, el flujo plástico del acero AISI 1045 es lento a partir de la primera carga aplicada, ya que el material ofrecerá mayor resistencia a ser deformado en la medida que el mismo se endurece superficialmente.

Deformación logarítmica $\varepsilon$ frente a las fuerzas aplicadas

La deformación presenta variaciones en todo su trayecto respecto a las fuerzas aplicadas como se observa en la figura 4.

Como se aprecia en la figura existen diferentes tendencias en las curvas de deformación, hay una tendencia a disminuir desde un $0,12 \%$ hasta un $0,06 \%$ cuando se aplica fuerza de $2500 \mathrm{~N}$, este efecto está condicionado porque en la medida que el material endurece presenta mayor resistencia a ser deformado, esta actitud en el acero AISI 1045 está motivado a que el mismo presenta buenas condiciones para ser deformado superficialmente y endurecer en frío. 


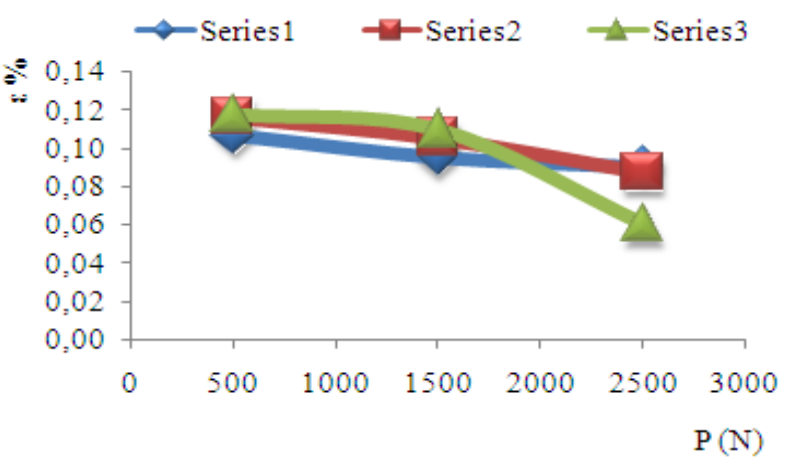

Figura 4. Comportamiento de la deformación frente a las fuerzas aplicadas.

\section{Deformación real vs fuerza $F$}

El \% de deformación verdadera $(\varepsilon)$ depende de la capacidad de deformar que presente el material, además de que también tiene una dependencia de la deformación ingenieril e.

La figura 5 establece el comportamiento de la deformación verdadera frente a la fuerza.

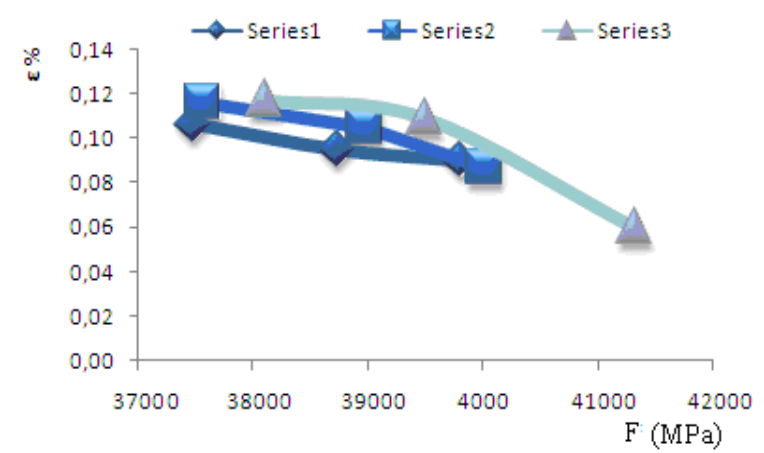

Figura 5. Comportamiento de la deformación verdadera $\varepsilon$ frente a la fuerza $\mathrm{F}$.

Se puede observar a medida que aumenta la fuerza disminuye el \% de deformación, este comportamiento viene influenciado porque cuando el material se deforma, mayor resistencia va a ofrecer este a ser deformado y por tanto menor va a ser la capacidad de deformación.

Comportamiento de la deformación verdadera frente al esfuerzo verdadero

La figura 6 muestra la deformación verdadera respecto al esfuerzo verdadero.

En este caso se puede observar el comportamiento de la curva tenso - deformacional, pero ya analizada para su modelo real de la forma $\mathrm{y}=\mathrm{ax} 2$, que es la forma de la ecuación de Hollomon $\sigma=K . \varepsilon n$, la cual rige todo el procedimiento de cálculo para determinar los parámetros de dureza del acero AISI 1045 deformado por el método de la rodadura.

Las deformaciones $(\varepsilon)$ decrecen con el aumento del esfuerzo $(\sigma)$, mientras mayor dureza adquiera el material en frío, más grande será la fuerza que debe ejercer la máquina. Esto ocurre porque a medida que el material endurece menor va a ser la elongación ingenieril. La diferencia entre las series viene dada porque a cada serie se le aplicaron avances y números de revoluciones distintos, parámetros estos que no tienen una influencia homogénea en todo el proceso.

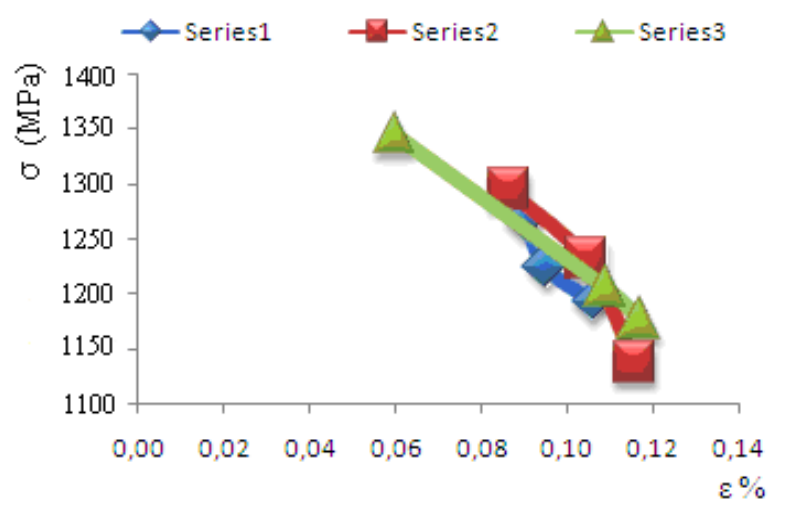

Figura 6. Comportamiento de la deformación verdadera respecto al esfuerzo verdadero

No obstante este $\%$ de deformación tiene un valor de 0,1 $\%$, en relación con los experimentos antes realizados con el acero AISI 1045 sometidos a otros tratamientos y sin tratamiento alguno, se puede observar que la deformación prácticamente no varía con el empleo del tratamiento sea cual fuere este, no siendo así con el esfuerzo, que aumenta con la deformación superficial del material y con la fuerza aplicada.

\section{CONCLUSIONES}

La ecuación de Hollomon, permitió determinar la curva esfuerzo-deformación del material trabajado en frío, debido a que se ajustó al comportamiento del material luego de ser endurecido y traccionado.

Los resultados demuestran que el coeficiente $\mathrm{n}$ nos puede dar la descripción de los parámetros de la deformación en cualquier punto, o sea, con cualquier carga, dentro de los parámetros establecidos. 
Los valores del coeficiente de endurecimiento obtenido para cada fuerza, avance y número de revoluciones, permiten establecer que, estas variables tienen incidencias significativas durante el proceso de deformación en frío del acero AISI 1045.

\section{AGRADECIMIENTOS}

Al Laboratorio de Resistencia de Materiales del Instituto Superior Minero metalúrgico de Moa, por la colaboración brindada en la realización de los ensayos.

\section{NOTACIÓN}

$\begin{array}{lll}\sigma- & \text { Esfuerzo real, MPa. } \\ \varepsilon- & \begin{array}{l}\text { Deformación real } \\ \mathrm{K}-\end{array} & \begin{array}{l}\text { Constante que define el coeficiente de } \\ \text { endurecimiento }\end{array} \\ \mathrm{n}- & \begin{array}{l}\text { Exponente del endurecimiento. } \\ \mathrm{S}-\end{array} & \\ \mathrm{tensión} \mathrm{ingenieril.} & & \\ \mathrm{ln}- & \text { logaritmo natural en las condiciones de } \\ \text { establecimiento de la curva } & & \\ \mathrm{e}- & \text { deformación ingenieril }\end{array}$

\section{REFERENCIAS}

Altenberger, I. (2003) Deep rolling - the past, the present and the future. University of Kassel. Institute of Materials Engineering. Monchebergstrasse 3, 34125, Germany.

Aparicio. G, D' Armas, H, Ciaccia, M. (2007) Comportamiento elastoplástico en tracción de láminas de acero ASTM A-569. Revista Ingeniería UC. Vol. 14, No 1, 57-63

Callister, W. (1999) Materials Science and Engineering An Introduction Fifth Edition Department of Metallurgical Engineering University of Utah. John Wiley \& Sons, Inc. 8195 pp. ISBN $0-471-32013-7$

Goicolea, J., Gabaldón, F. y García-Garino, C. (1996) Interpretación de la estricción en el ensayo de tracción empleando modelos hipoelásticos e hiperelásticos eds., Memorias del III Congreso de Métodos Numéricos en Ingeniería. SEMNI, Zaragoza, 1996.
González-Castellanos, R., Principios básicos de escalado. Edición electrónica, Ed. Universitaria, La Habana, Cuba. 2000.

García-Garino, C., Mirasso, A., Raichman, S. y Goicolea, J. M. (1999) Análisis de sensibilidad local y unicidad aparente en el problema de estricción de barras metálicas eds Mecánica Computacional, Págs. 281-288.

García-Mateo, C; Romero, J.L y Rodríguez-Ibabe, J.M. (1998) Efecto del Vanadio en la Forja en Tibio de un Acero de Medio Carbono. Rev. Metal Madrid, 34 Mayo. p. 253 - 257.

Hill R. (1998) The Mathematical theory of plasticity Clarendon Press, Oxford

Hollomon. (1945) Tensil Deformation, Trans. AIME, Vol. 162, pp 268.

Jonson W. and Mellor P. B. Engineering Plasticity. Van Nostrand Reinhold Editorial, London. 1973

Low. (1949) Properties of Engineering Materials, ASM.

Ludwik. P (1909) Elemente der Technologischen Mechanik, Springer Verlag OHG, Berlin.

Norma ASTM E 646 - 00: Standard Test Method for Tensile Strain - Hardening Exponents (n-Values) of Metallic Sheet Materials.

Norris, D., Moran, B., Scudder, J. y Quiñones, D. A (1978) computer simulation of the tension test. Journal of Mechanics, Physics and Solids, tomo26. Págs. 119, 1978

Novillo. E. D, Picasso. A., Cuniberti. A. (1999) Envejecimiento por deformación en un acero AISI 316. Anales SAM. Vol. 17, No. 4 pp. 95 - 100. 1999.

Ono K. (1972) Strain - Hardening Equations and Uniform Strain, Metallurgical Transactions, Vol. 3, pp. $749-751$.

Ortiz, M. y Popov, E. (1983) Distorsional hardening rules for metal plasticity. Journal of Engineering Mechanics, tomo109, no 4: págs.1042 - 1057.

Valiente, A. (2001) On Bridgman's stress solution for a tensile neck applied to axisymmetrical blunt notched 
tension bars. Journal of Applied Mechanics, tomo 68: Págs. $412-419$.

Voce. (1948). The Relationship between Stress and Strain for Homogeneous Deformation J. Inst. Met., Vol. 74, pp 537-562.

Zlokarnik, M., Scale - up in chemical engineering. Ed. Wiley - VCH Verlag GmbH. Weinheim, Germany. 2002.

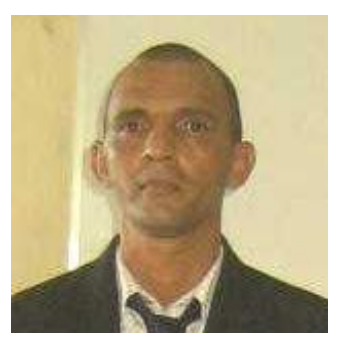

Tomás Fernández Columbié, graduado de profesor en la Educación Técnica Profesional, en el Instituto Técnico de Holguín, 1986, Licenciado en Construcción de Maquinaria en el Instituto Superior Pedagógico de Santiago de Cuba, 1992. Master en Ciencias en Electromecánica del Instituto Superior Minero Metalúrgico de Moa. Área de investigación, materiales. Profesor asistente del departamento de Mecánica. 